\title{
ABOUT THE LINDEBERG METHOD FOR STRONGLY MIXING SEQUENCES
}

\author{
EMMANUEL RIO
}

\begin{abstract}
We extend the Lindeberg method for the central limit theorem to strongly mixing sequences. Here we obtain a generalization of the central limit theorem of Doukhan, Massart and Rio to nonstationary strongly mixing triangular arrays. The method also provides estimates of the Lévy distance between the distribution of the normalized sum and the standard normal.
\end{abstract}

\section{INTRODUCTION}

In this paper, we are interested in the central limit theorem for strongly mixing and possibly nonstationary sequences of real-valued random variables. First let us recall some recent results for strongly mixing sequences, improving on the classical results of Ibragimov (1962). In order to state these results, we need some more notation.

DeFinition 1. Let $\left(X_{i}\right)_{i \in \mathbb{Z}}$ be a sequence of real-valued random variables with mean zero. For any nonincreasing cadlag function $H: \mathbb{R}^{+} \rightarrow \mathbb{R}^{+}$, let $H^{-1}$ denote the cadlag inverse function of $H$, which is defined by

$$
H^{-1}(u)=\sup \left\{t \in \mathbb{R}^{+}: H(t)>u\right\},
$$

with the convention that $\sup \emptyset=0$. For any real-valued random variable $X$ with distribution function $F$, we denote by $Q_{X}$ or $Q_{F}$ the inverse function of $t \rightarrow \mathbb{P}(|X|>t)$. We set $Q_{i}=Q_{X_{i}}$.

If $\left(u_{n}\right)_{n \geq 0}$ is a nonincreasing sequence of nonnegative real numbers, we denote by $u($.$) the cadlag rate function which is defined by u(t)=u_{[t]}$. Throughout the sequel, $u^{-1}$ denotes the inverse function of this rate function $u($.$) .$

It comes from Doukhan, Massart and Rio (1994) that the central limit theorem for strictly stationary sequences with strong mixing coefficients $\left(\alpha_{n}\right)_{n \geq 0}$ holds under the integral condition

$$
\int_{0}^{1} \alpha^{-1}(x) Q_{0}^{2}(x) d x<\infty .
$$

ESAIM: Probability and Statistics is an electronic journal with URL address http://www.emath.fr/ps.

Received by the journal 1 February 1995. Revised 16 October. Accepted for publication 11 December 1995 
When $X_{0}$ satisfies the moment assumption $\mathbb{E}\left(\left|X_{0}\right|^{r}\right)<\infty$, condition (I.1) holds as soon as

$$
\sum_{n>0} n^{2 /(r-2)} \alpha_{n}<\infty
$$

(I.2) improves on Ibragimov's condition $\sum_{n>0} \alpha_{n}^{r /(r-2)}<\infty$. Moreover, from a paper of Bolthausen (1980) dealing with rates of convergence in the central limit theorem for Markov chains on a countable space, we believe that (I.2) cannot be improved for strongly mixing Markov chains.

The aim of this paper is, first to extend the central limit theorem for strongly mixing sequences to a central limit theorem for triangular arrays, and second to obtain rates of convergence in the central limit theorem. We refer the reader to Bergström (1972), Krieger (1984) and Samur (1984) for the central limit theorem for $\phi$-mixing triangular arrays with stationary rows and to Tikhomirov (1980) and Götze and Hipp (1983) for rates of convergence and asymptotic expansions in the central limit theorem for mixing sequences. Let us also mention the recent works of Peligrad and Utev (1994) and Peligrad (1995), which improve the previous results for triangular arrays.

The proofs of central limit theorems for mixing sequences often are based either on Gordin's theorem (1969) [see Hall and Heyde (1980)] or on the Bernstein's method [see Ibragimov and Linnik (1971)]. Unfortunately the extension of these techniques to nonstationary sequences is quite delicate. So, in order to obtain central limit theorems for triangular arrays, we will adapt the Lindeberg method [see Lindeberg 1922] to strongly mixing sequences. Up to our knowledge, the Lindeberg method was first used in the setting of strongly mixing processes by Doukhan and Portal (1983a) [see also Doukhan and Portal (1987)]. They studied the rates of convergence in the multidimensional central limit theorem and extended some estimates of Yurinskii (1977) to mixing sequences. Next Doukhan, León and Portal [(1984) and (1985)] obtained some related results for Hilbert space valued stationary mixing random variables. This method has two main advantages: it leads to optimal conditions concerning the tail ditributions of the random variables and it gives precise estimates of the Lévy distance between the distribution of the normalized sum and the standard normal distribution for stationary and strongly mixing sequences.

Let us now recall the Lindeberg central limit theorem for independent summands. Let $\left(X_{i n}\right)_{i \in[1, n]}$ be a triangular array of independent squareintegrable random variables with mean zero, normalized in such a way that $\operatorname{Var}\left(X_{1 n}+\cdots+X_{n n}\right)=1$. Let $S_{n n}=X_{1 n}+\cdots+X_{n n}$. Then $S_{n n}$ converges in distribution to a standard normal distribution if, for any positive $\varepsilon$,

$$
\sum_{i=1}^{n} \mathbb{E}\left(X_{i n}^{2} \mathbb{I}_{\left|X_{i n}\right|>\varepsilon}\right) \rightarrow 0 \text { as } n \rightarrow \infty,
$$

which is equivalent to

$$
\sum_{i=1}^{n} \int_{0}^{1} Q_{X_{i n}}^{2}(x)\left(Q_{X_{i n}}(x) \wedge 1\right) d x \rightarrow 0 \text { as } n \rightarrow \infty .
$$


In a recent note concerning moment inequalities for stationary strongly mixing sequences [see Rio (1994)], we prove that, for any $p \geq 2$, the moment condition

$$
M_{p, \alpha}\left(Q_{0}\right)=\int_{0}^{1 / 2}\left(\alpha^{-1}(x / 2) Q_{0}(x)\right)^{p} \frac{d x}{\alpha^{-1}(x / 2)}<\infty
$$

is sufficient to imply a Rosenthal type inequality of order $p$ [see Rosenthal (1970) for these inequalities in the independent setting]. Hence we obtain a generalization of the classical moment inequalities by replacing $Q_{0}$ by $\alpha^{-1}(x / 2) Q_{0}(x)$ and the Lebesgue measure by the weighted measure $d x / \alpha^{-1}(x / 2)$. Exactly in the same way, we will obtain a generalization of the Lindeberg condition to strongly mixing sequences by replacing $Q_{X_{i n}}$ by $\alpha^{-1}(x / 2) Q_{X_{i n}}$ and $d x$ by $d x / \alpha^{-1}(x / 2)$ in (I.3). Since the Lindeberg method provides estimates of the error between the characteristic function of the normalized sum and the characteristic function of the standard normal, we also obtain upper bounds on the Lévy distance between the distributions functions in the stationary case, via Esseen's inequality (1945). In particular, if $M_{2+\delta, \alpha}\left(Q_{0}\right)$ is finite for some $\delta \leq(\sqrt{5}-1) / 2$, we obain an upper bound of the order of $n^{-\delta / 2}$.

\section{THE MAIN RESUlts}

Definition 2. For any two $\sigma$-algebras $\mathcal{A}$ and $\mathcal{B}$ in $(\Omega, \mathcal{T}, \mathbb{P})$, let

$$
\alpha(\mathcal{A}, \mathcal{B})=\sup _{(A, B) \in \mathcal{A} \times \mathcal{B}}|\mathbb{P}(A \cap B)-\mathbb{P}(A) \mathbb{P}(B)|
$$

denote the strong mixing coefficient introduced by Rosenblatt (1956). The strong mixing coefficients $\left(\alpha_{n}\right)_{n>0}$ of the sequence $\left(X_{i}\right)_{i \in \mathbb{Z}}$ are defined by

$$
\alpha_{n}=\sup _{k \in \mathbb{Z}} \alpha\left(\mathcal{F}_{k-n}, \mathcal{G}_{k}\right)
$$

where $\mathcal{F}_{l}=\sigma\left(X_{i}: i \leq l\right)$ and $\mathcal{G}_{l}=\sigma\left(X_{i}: i \geq l\right)$. We make the convention that $\alpha_{0}=1 / 4$.

Throughout the section, $Q$ is any nonincreasing function from $[0,1]$ into $\mathbb{R}^{+}$such that $Q \geq \sup _{i>0} Q_{i}$.

Let us recall some basic covariance inequalities for strongly mixing sequences, improving on the covariance inequalities of Davydov (1968). By Theorems 1.1 and 1.2 in Rio (1993), the following upper bounds on the variance of the partial sums of strongly mixing sequences hold.

Proposition 1. Let $\left(X_{i}\right)_{i \in \mathbb{Z}}$ be a sequence of real-valued random variables with finite variance and mean zero. Let the strong mixing coefficients $\left(\alpha_{n}\right)_{n \geq 0}$ be defined by $\alpha_{0}=1 / 4$ and

$$
\alpha_{n}=\sup _{k \in \mathbb{Z}} \alpha\left(\mathcal{F}_{k-n}, \sigma\left(X_{k}\right)\right)
$$


for any positive $n$. Then, for any integers $s$ and $t$ such that $s<t$,

$$
\left|\operatorname{Cov}\left(X_{s}, X_{t}\right)\right| \leq 2 \int_{0}^{2 \alpha_{t-s}} Q_{t}(x) Q_{s}(x) d x .
$$

Let $S_{n}=X_{1}+\cdots+X_{n}$. Then

$$
\operatorname{Var} S_{n} \leq \sum_{(s, t) \in] 0, n]^{2}}\left|\operatorname{Cov}\left(X_{s}, X_{t}\right)\right| \leq 4 \sum_{i=1}^{n} \int_{0}^{1 / 2}\left[\alpha^{-1}(x / 2) \wedge n\right] Q_{i}^{2}(x) d x,(b)
$$

where $\alpha^{-1}$ denotes the inverse of the mixing rate function associated with the strong mixing coefficients $\left(\alpha_{n}\right)_{n \geq 0}$.

Suppose that

$$
M_{2, \alpha}(Q)=\int_{0}^{1 / 2} \alpha^{-1}(x / 2) Q^{2}(x) d x<+\infty .
$$

Then

$$
n^{-1} \operatorname{Var} S_{n} \leq 4 M_{2, \alpha}(Q) .
$$

Hence, if $\left(X_{i}\right)_{i \in \mathbb{Z}}$ is strictly stationary, the series $\sum_{t \in \mathbb{Z}} \operatorname{Cov}\left(X_{0}, X_{t}\right)$ is absolutely convergent to some non negative number $\sigma^{2}$,

$$
\lim _{n \rightarrow+\infty} n^{-1} \operatorname{Var} S_{n}=\sigma^{2} \text { and } \sigma^{2} \leq \sum_{t \in \mathbb{Z}}\left|\operatorname{Cov}\left(X_{0}, X_{t}\right)\right| \leq 4 M_{2, \alpha}(Q) .
$$

The main results are the following estimates of the nearness of the characteristic function of the normalized sum and of the characteristic function of the standard normal.

Theorem 1. Let $\left(X_{i}\right)_{i \in \mathbb{Z}}$ be a strongly mixing sequence of real-valued random variables with finite variance and mean zero. Suppose that $X_{i}=0$ a.s. for any $i \notin[1, n]$. Let $\varphi_{k}(t)=\mathbb{E}\left(\exp \left(i t S_{k}\right)\right)$,

$$
V_{0}=0, V_{k}=\operatorname{Var} S_{k} \text { and } V_{n}^{*}=\sup _{k \in[1, n]} V_{k} .
$$

(i) For any nonnegative quantile function $Q$ and any positive $t$, let

$$
M_{3, \alpha}(Q, t)=\int_{0}^{1} \alpha^{-1}(x / 2) Q^{2}(x)\left(t \alpha^{-1}(x / 2) Q(x) \wedge 1\right) d x .
$$

Then, for any real $t$,

$$
\left|\exp \left(V_{n} t^{2} / 2\right) \varphi_{n}(t)-1\right| \leq 58 t^{2} \exp \left(V_{n}^{*} t^{2} / 2\right) \sum_{k=1}^{n} M_{3, \alpha}\left(Q_{k},|t|\right) .
$$

(ii) Suppose furthermore that $\left(X_{i}\right)_{i \in \mathbb{Z}}$ fulfills condition (1.3). Then, for any real $t$

$$
\left|\exp \left(V_{n} t^{2} / 2\right) \varphi_{n}(t)-1\right| \leq 8(\sqrt{2}+1) t^{2} M_{3, \alpha}(Q,|t|) \sum_{k=0}^{n} \exp \left(V_{k} t^{2} / 2\right) .
$$


Remark 1. It comes from Proposition 2 in section 2 that Theorem 1 and Corollary 1 may be obtained under the following weaker definition of the strong mixing coefficients:

$$
\alpha_{n}=\sup _{k \in \mathbb{Z}} \sup _{\left(p_{1}, p_{2}\right) \in \mathbb{N}^{2}} \alpha\left(\mathcal{F}_{k-n}, \sigma\left(X_{k+p_{1}}, X_{k+p_{2}}\right)\right) .
$$

Consequently some upper bounds on these mixing coefficients would be of interest.

(i) is a result generalizing Lindeberg's one [see Lindeberg (1922)] to strongly mixing sequences. So, our main application of (i) is the following central limit theorem for strongly mixing triangular arrays.

Corollary 1. Let $\left(X_{i n}\right)_{n>0, i \in[1, n]}$ be a double array of real-valued random variables with finite variance and mean zero. Let $\left(\alpha_{k, n}\right)_{k>0}$ be the sequence of strong mixing coefficients of the sequence $\left(X_{i n}\right)_{i \in[1, n]}$ and $\alpha_{(n)}^{-1}$ be the inverse function of the the associated mixing rate function. We set

$$
S_{i n}=X_{1 n}+\cdots+X_{i n} \text { and } V_{i, n}=\operatorname{Var} S_{i n} .
$$

Suppose furthermore that

$$
\limsup _{n \rightarrow \infty} \max _{i \in[1, n]}\left(V_{i, n} / V_{n, n}\right)<\infty
$$

Let $Q_{i, n}=Q_{X_{i n}}$. Then $S_{n n}$ converges to the standard normal distribution if

$$
V_{n, n}^{-3 / 2} \sum_{i=1}^{n} \int_{0}^{1} \alpha_{(n)}^{-1}(x / 2) Q_{i, n}^{2}(x) \inf \left(\alpha_{(n)}^{-1}(x / 2) Q_{i, n}(x), \sqrt{V_{n, n}}\right) d x \longrightarrow 0
$$

as $n$ tends to $\infty$.

REMARK 2. Note that $\alpha_{k, n}=0$ for amy $k \geq n$, which implies that $\alpha_{(n)}^{-1}(x / 2) \leq n$ for any positive $x$.

Application 1. Let $\left(\xi_{i}\right)_{i \in \mathbb{Z}}$ be a strictly stationary and strongly mixing sequence of real-valued random variables with mean zero, satisfying the condition $M_{2, \alpha}\left(Q_{\xi_{0}}\right)<\infty$. Let $\left(a_{i n}\right)_{i \in[1, n]}$ be a triangular array of real numbers such that

$$
\sum_{i=1}^{n} a_{i n}^{2}=1 \text { and } \lim _{n \rightarrow \infty} \max _{i \in[1, n]}\left|a_{i n}\right|=0
$$

We set $X_{i n}=a_{i n} \xi_{i}$. Then, by (b) of Proposition 1,

$$
\operatorname{Var} S_{k n} \leq 4 \sum_{i=1}^{n} a_{i n}^{2} M_{2, \alpha}\left(Q_{\xi_{i}}\right) \leq 4 M_{2, \alpha}\left(Q_{\xi_{0}}\right)
$$


This inequality ensures that Corollary 1 (a) is equivalent to $\lim \inf _{n} V_{n, n}>0$. Consequently, if (a) holds, condition (b) of Corollary 1 is ensured by the mixing condition

$$
\sum_{k=1}^{n} a_{k n}^{2} \int_{0}^{1} \alpha^{-1}(x / 2) Q_{\xi_{0}}^{2}(x)\left(\left|a_{k n}\right| \alpha^{-1}(x / 2) Q_{\xi_{0}}(x) \wedge 1\right) d x \longrightarrow 0,
$$

as $n \rightarrow \infty$, where $\alpha^{-1}$ stands for the inverse function of the strong mixing rate function of $\left(\xi_{i}\right)_{i \in \mathbb{Z}}$. Since $\max _{i \in[1, n]}\left|a_{i n}\right|$ tends to zero as $n \rightarrow \infty$, Lebesgue dominated convergence theorem lets us prove that the above expression converges to zero. Hence the CLT for $S_{n n}$ holds, which generalizes (c) of Theorem 2.2 of Peligrad and Utev (1994).

Let us now give some applications of Theorem 1(ii) to Berry-Esseen type estimates. Let the class $\Phi$ of two times differentiable Orlicz functions be defined by :

$$
\begin{array}{r}
\Phi=\left\{\phi: \mathbb{R}^{+} \rightarrow \mathbb{R}^{+} ; \phi \text { convex, } \phi(0)=\phi^{\prime}(0)=0\right. \\
\left.\phi^{\prime \prime} \text { nondecreasing, concave }\right\} .
\end{array}
$$

For any $\phi$ in $\Phi$, we define the weighted moments $M_{\phi, \alpha}(Q)$ by

$$
M_{\phi, \alpha}(Q)=\int_{0}^{1 / 2} \frac{\phi\left(\alpha^{-1}(x / 2) Q(x)\right)}{\alpha^{-1}(x / 2)} d x
$$

If $\phi(x)=x^{r}$, we set $M_{\phi, \alpha}(Q)=M_{r, \alpha}(Q)$.

When $\left(X_{i}\right)_{i \in \mathbb{Z}}$ is a strictly stationary sequence verifying (1.3) and the additional condition

$$
\sigma^{2}=\sum_{t \in \mathbb{Z}} \operatorname{Cov}\left(X_{0}, X_{t}\right) \neq 0
$$

the central limit theorem holds [see Doukhan, Massart and Rio (1994)]. More precisely $S_{n} / \sigma \sqrt{n}$ converges to a standard normal distribution. We then get the following estimates of the Lévy distance for partial sums of a stationary sequence as a by-product of Theorem 1(ii).

Theorem 2. Let $\left(X_{i}\right)_{i \in \mathbb{Z}}$ be a strictly stationary sequence of real-valued random variables with mean zero and finite variance satisfying (1.3) and (1.8). Let the sequence of strong mixing coefficients be defined by (1.2). Let $\Psi$ denote the d.f. of a standard normal.

(i) For any $\delta$ in $] 0,1[$ such that

$$
\Lambda_{2+\delta, \alpha}(Q)=\sup _{x \in] 0,1 / 4[} \frac{x}{\alpha^{-1}(x)}\left(\alpha^{-1}(x) Q(x)\right)^{2+\delta}<\infty,
$$

we have:

$$
\Pi_{n}=\sup _{x \in \mathbb{R}}\left|\mathbb{P}\left(S_{n} \leq x \sigma \sqrt{n}\right)-\Psi(x)\right|=O\left(n^{-\delta / 2} \vee n^{-(1+\delta) /(4+2 \delta)}\right) .
$$


(ii) Suppose that $M_{3, \alpha}(Q)<\infty$. Then

$$
\sup _{x \in \mathbb{R}}\left|\mathbb{P}\left(S_{n} \leq x \sigma \sqrt{n}\right)-\Psi(x)\right|=O\left(n^{-1 / 3}\right) .
$$

(iii) Suppose that $M_{\phi, \alpha}(Q)<\infty$ for some function $\phi$ in $\Phi$ verifying $\phi(x) \sim$ $x^{2}(\log x)^{p}$ for some positive $p$ as $x \rightarrow+\infty$. Then

$$
\sup _{x \in \mathbb{R}}\left|\mathbb{P}\left(S_{n} \leq x \sigma \sqrt{n}\right)-\Psi(x)\right|=O\left((\log n)^{-p}\right) .
$$

REMARK 2. Note that the moment condition $M_{2+\delta, \alpha}(Q)<\infty$ is stronger than (1.9). Moreover it comes from the lower bouds of Tikhomirov (1980) that (i) of Theorem 2 is nearly optimal when $\delta \leq(-1+\sqrt{5}) / 2$.

\section{Application 2.}

(a) Bounded random variables. Assume that $\left\|X_{0}\right\|_{\infty}<\infty$. Then (1.9) holds if and only if $\alpha_{n}=O\left(n^{-1-\delta}\right)$. In that case, Theorem 2(i) yields $\Pi_{n}=O\left(n^{-\delta / 2}\right)$ if $\delta \leq(-1+\sqrt{5}) / 2$ and $\Pi_{n}=O\left(n^{-(1+\delta) /(4+2 \delta)}\right)$ otherwise, which improves on Theorem 1 of Tikhomirov (1980). Now $M_{3, \alpha}(Q)$ is finite if and only if $\sum_{n>0} n \alpha_{n}<\infty$. Then, by (ii) of Theorem $2, \Pi_{n}=O\left(n^{-1 / 3}\right)$.

Under the weaker condition $M_{\phi, \alpha}(Q)<\infty$ for some $\phi$ in $\Phi$ such that $\phi(x) \sim x^{2}(\log x)^{p}$, Theorem 2(iii) yields $\Pi_{n}=O\left((\log n)^{-p}\right)$ under the condition $\sum_{n>0}(\log n)^{p} \alpha_{n}<\infty$. Note that the loss between this condition and Ibragimov's condition for the central limit theorem $\sum_{n>0} \alpha_{n}<\infty$ [see Ibragimov and Linnik (1971) for the CLT] is logarithmic.

(b) Conditions on the tail function. Suppose that, for some $\rho>2$, $\mathbb{P}\left(\left|X_{0}\right|>u\right)=O\left(u^{-\rho}\right)$. Then

$$
\Lambda_{\rho}(Q)=\sup _{u \in] 0,1]} u^{1 / \rho} Q(u)<\infty
$$

and $\Lambda_{2+\delta, \alpha}(Q)$ is finite for $\delta<\rho-2$ if $\alpha_{n}=O\left(n^{-\rho(1+\delta) /(\rho-2-\delta)}\right)$, while the condition $M_{3, \alpha}(Q)<\infty$ needs $\rho>3$ and the summability condition $\sum_{n>0} n \alpha_{n}^{1-3 / \rho}<\infty$.

(c) Moment conditions. Suppose that, for some $r>3, \mathbb{E}\left(\left|X_{0}\right|^{r}\right)<\infty$. Then, by the Hölder inequality applied on $[0,1], M_{3, \alpha}(Q)$ is finite if

$$
\sum_{n>0} n^{(r+3) /(r-3)} \alpha_{n}<\infty
$$

Under this mixing condition, Theorem 2(ii) yields $\Pi_{n}=O\left(n^{-1 / 3}\right)$. However, Bolthausen (1980 and 1982) obtains $\Pi_{n}=O\left(n^{-1 / 2}\right)$ for Harris recurrent Markov chains under the same mixing condition.

(d) Exponential mixing rates. Assume that the mixing coefficients satisfy $\alpha_{n}=O\left(a^{n}\right)$ for some $a$ in $] 0,1[$. Then Theorem 2 in Tikhomirov (1980) yields

$$
\Pi_{n}=O\left(n^{-\delta / 2}(\log n)^{1+\delta}\right)
$$


under the moment assumption $\mathbb{E}\left(\left|X_{0}\right|^{2+\delta}\right)<\infty$, for any $\left.\left.\delta \in\right] 0,1\right]$. If $\delta<1$, one can use the Stein-Tikhomirov method to obtain $\Pi_{n}=O\left(n^{-\delta / 2}\right)$ under the moment condition

$$
\mathbb{E}\left(\left|X_{0}\right|^{2+\delta}\left(\log _{+}\left|X_{0}\right|\right)^{1+\delta}\right)<\infty .
$$

For geometric rates of mixing, (1.10) is equivalent to $M_{2+\alpha, \delta}(Q)<\infty$. Since this condition is stronger than (1.9), (i) of Theorem 2 slightly improves on the Stein-Tikhomirov method if $\delta \leq(\sqrt{5}-1) / 2$. However, Theorem 2 of Tikhomirov (1980) yields much more better rates if $\delta>(\sqrt{5}-1) / 2$.

For geometric rates of mixing, the condition $\mathbb{E}\left(X^{2}\left(\log _{+}|X|\right)^{p}\right)<\infty$ is equivalent to the condition $M_{\phi, \alpha}(Q)<\infty$ of (iii) with $\phi(x) \sim x^{2}(\log x)^{p-1}$. Thus (iii) of Theorem 2 yields $\Pi_{n}=O\left((\log n)^{1-p}\right)$, which improves on Corollaire 1 of Bulinskii and Doukhan (1990) in the special case of sequences.

\section{The Lindeberg METHOD For Strongly MiXing SEQUENCES.}

In this section, we generalize the Lindeberg method to strongly mixing sequences. The main step of this extension is Proposition 2 below. This proposition is applied to obtain estimates of the characteristic function of a sum of mixing random variables. In section 4 , we give an application of this proposition to moment inequalities for sums of non identically distributed random variables.

DEFinition 3. Let $\mathcal{F}\left(b_{2}, b_{3}\right)$ be the class of real-valued two times continuously differentiable functions $f$ such that $\left\|f^{(2)}\right\|_{\infty} \leq b_{2}$ and $\left\|f^{(2)}\right\|_{L} \leq b_{3}$, where $\left\|f^{(i)}\right\|_{\infty}=\sup _{x \in \mathbb{R}}\left|f^{(i)}(x)\right|$ and

$$
\left\|f^{(i)}\right\|_{L}=\sup _{\substack{(x, y) \in \mathbb{R}^{2} \\ x \neq y}} \frac{|f(x)-f(y)|}{|x-y|} .
$$

Let

$$
v_{k}=V_{k}-V_{k-1}=\mathbb{E}\left(X_{k}^{2}\right)+2 \sum_{i=1}^{k-1} \mathbb{E}\left(X_{k} X_{i}\right) .
$$

(In the weak dependence setting, $v_{k}$ may fail to be nonnegative). We set

$$
\Delta_{1, k}=\sup _{f \in \mathcal{F}\left(b_{2}, b_{3}\right)}\left|\mathbb{E}\left(f\left(S_{k-1}+X_{k}\right)-f\left(S_{k-1}\right)-\frac{v_{k}}{2} f^{\prime \prime}\left(S_{k-1}\right)\right)\right| .
$$

The main step of the proof of Theorem 1 is the following upper bound for $\Delta_{1, k}$.

Proposition 2. Let $\left(X_{i}\right)_{i \in \mathbb{Z}}$ be a sequence of real-valued random variables with finite variance and mean zero. Suppose that $X_{i}=0$ a.s. for any $i \leq 0$. Let the sequence $\left(\alpha_{n}\right)_{n \geq 0}$ of strong mixing coefficients of $\left(X_{i}\right)_{i \in \mathbb{Z}}$ be defined by (1.6). Let $u$ be any real in $[0,1 / 2]$ and $p=\alpha^{-1}(u / 2)$. We set

$$
M_{k}(x)=\sum_{i=0}^{k-1} Q_{k-i}(x) \mathbb{1}_{x<2 \alpha_{i}}
$$


and

$$
\left.M_{k}^{\prime}(x, u)\right)=\sum_{i=0}^{p-1} \sum_{l=0}^{p-1} Q_{k-i}(x) Q_{k-i-l}(x) \mathbb{I}_{x<2\left(\alpha_{i} \wedge \alpha_{l}\right)}
$$

Then

$$
\Delta_{1, k} \leq 4 b_{2} \int_{0}^{u} M_{k}(x) Q_{k}(x) d x+4 b_{3} \int_{0}^{1} M_{k}^{\prime}(x, u) Q_{k}(x \vee u) d x
$$

and

$$
\sum_{k=1}^{n} \Delta_{1, k} \leq 24 \sum_{k=1}^{n} \int_{0}^{1}\left[\alpha^{-1}(x / 2) \wedge n\right] Q_{k}^{2}(x) \inf \left(b_{2}, b_{3}\left[\alpha^{-1}(x / 2) \wedge n\right] Q_{k}(x)\right) d x .
$$

Proof. Throughout the proof, we make the convention that $S_{i}=0$ for any $i \leq 0$. We set

$$
M_{k}(x, u)=\sum_{i=0}^{p-1} Q_{k-i}(x) \mathbb{I}_{x<2 \alpha_{i}} \text { and } \bar{X}_{k}=\left(X_{k} \wedge Q_{k}(u)\right) \vee\left(-Q_{k}(u)\right) .
$$

We start by the proof of (a). By the Taylor integral formula,

$$
\begin{aligned}
f\left(S_{k}\right)-f\left(S_{k-1}\right)-f^{\prime}\left(S_{k-1}\right) X_{k} & =X_{k} \int_{0}^{1}\left(f^{\prime}\left(S_{k-1}+v X_{k}\right)-f^{\prime}\left(S_{k-1}\right)\right) d v \\
& =X_{k} \int_{0}^{1}\left(f^{\prime}\left(S_{k-1}+v X_{k}\right)-f^{\prime}\left(S_{k-1}+v \bar{X}_{k}\right)\right) d v \\
& +X_{k} \bar{X}_{k} \int_{0}^{1} \int_{0}^{1} v f^{\prime \prime}\left(S_{k-1}+v v^{\prime} \bar{X}_{k}\right) d v d v^{\prime}
\end{aligned}
$$

The first term on right hand is bounded up by $b_{2}\left|X_{k}\left(X_{k}-\bar{X}_{k}\right)\right| / 2$. Moreover

$$
\left|\int_{0}^{1} \int_{0}^{1} v f^{\prime \prime}\left(S_{k-1}+v v^{\prime} \bar{X}_{k}\right) d v d v^{\prime}-\frac{1}{2} f^{\prime \prime}\left(S_{k-1}\right)\right| \leq \frac{b_{3}}{6}\left|\bar{X}_{k}\right|
$$

Since

$$
\mathbb{E}\left|X_{k}\left(X_{k}-\bar{X}_{k}\right)\right|=\int_{0}^{u} Q_{k}(x)\left(Q_{k}(x)-Q_{k}(u)\right) d x,
$$

it follows that

$$
\begin{aligned}
& \left|\mathbb{E}\left(f\left(S_{k}\right)-f\left(S_{k-1}\right)-f^{\prime}\left(S_{k-1}\right) X_{k}-\frac{1}{2} f^{\prime \prime}\left(S_{k-1}\right) X_{k} \bar{X}_{k}\right)\right| \leq \\
& \frac{b_{2}}{2} \int_{0}^{u} Q_{k}(x)\left(Q_{k}(x)-Q_{k}(u)\right) d x+\frac{b_{3}}{3} \int_{0}^{1 / 2} Q_{k}^{2}(x) Q_{k}(x \vee u) d x .
\end{aligned}
$$

Now we control the second order term. Let $\Gamma_{k, i}=f^{\prime \prime}\left(S_{k-i}\right)-f^{\prime \prime}\left(S_{k-i-1}\right)$. Clearly

$$
f^{\prime \prime}\left(S_{k-1}\right) X_{k} \bar{X}_{k}=\sum_{i=1}^{p-1} \Gamma_{k, i} X_{k} \bar{X}_{k}+f^{\prime \prime}\left(S_{k-p}\right) X_{k} \bar{X}_{k} .
$$


Since $\left|\Gamma_{k, i}\right| \leq b_{3}\left|X_{k-i}\right|$, it follows from Proposition 1(a) applied to $X=\Gamma_{k, i}$ and $Y=X_{k} \bar{X}_{k}$ that

$$
\left|\operatorname{Cov}\left(\Gamma_{k, i}, X_{k} \bar{X}_{k}\right)\right| \leq 2 b_{3} \int_{0}^{2 \alpha_{i}} Q_{k-i}(x) Q_{k}(x) Q_{k}(x \vee u) d x .
$$

Noting that $2 \alpha_{p} \leq u$, we also get that

$$
\left|\operatorname{Cov}\left(f^{\prime \prime}\left(S_{k-p}\right), X_{k} \bar{X}_{k}\right)\right| \leq 2 b_{2} \int_{0}^{u} Q_{k}(x) Q_{k}(x \vee u) d x .
$$

Hence

$$
\begin{aligned}
& \frac{1}{2}\left|\operatorname{Cov}\left(f^{\prime \prime}\left(S_{k-1}\right), X_{k} \bar{X}_{k}\right)\right| \leq \\
& \quad \int_{0}^{1 / 2}\left(b_{3}\left(M_{k}(x, u)-Q_{k}(x)\right)+b_{2} \mathbb{I}_{x<u}\right) Q_{k}(x) Q_{k}(x \vee u) d x,
\end{aligned}
$$

which together with (2.5) and (2.4) implies that

$$
\begin{gathered}
\left|\mathbb{E}\left(f\left(S_{k-1}+X_{k}\right)-f\left(S_{k-1}\right)-f^{\prime}\left(S_{k-1}\right) X_{k}\right)-\frac{1}{2} \mathbb{E}\left(f^{\prime \prime}\left(S_{k-1}\right)\right) \mathbb{E}\left(X_{k}^{2}\right)\right| \leq \\
b_{2} \int_{0}^{u} Q_{k}^{2}(x) d x+b_{3} \int_{0}^{1} M_{k}(x, u) Q_{k}(x) Q_{k}(x \vee u) d x
\end{gathered}
$$

It remains to give an estimate of the expectation of $f^{\prime}\left(S_{k-1}\right) X_{k}$. Clearly

$$
\mathbb{E}\left(f^{\prime}\left(S_{k-1}\right) X_{k}\right)=\sum_{i=1}^{k-1} \operatorname{Cov}\left(f^{\prime}\left(S_{k-i}\right)-f^{\prime}\left(S_{k-i-1}\right), X_{k}\right) .
$$

In order to estimate the terms in (2.8), we need the following general principle, due to Fréchet $(1951,1957)$ and Bass (1955).

Lemma 1. Let $Z_{1}, \ldots Z_{m}$ be nonnegative random variables with respective quantile functions $Q_{Z_{1}}, \ldots, Q_{Z_{m}}$. Then

$$
\mathbb{E}\left(Z_{1} \ldots Z_{m}\right) \leq \int_{0}^{1} Q_{Z_{1}}(x) \ldots Q_{Z_{m}}(x) d x
$$

Remark 3. Actually Fréchet gives a complete proof in the case $m=2$. However, the proof uses the same arguments in the general case.

Let us also state the following by-products of Lemma 1, which will be used later on : with the same notations as in Lemma 1,

$$
\int_{0}^{1} Q_{Z_{1} Z_{2}}(x) Q_{Z_{3}}(x) \ldots Q_{Z_{m}}(x) d x \leq \int_{0}^{1} Q_{Z_{1}}(x) Q_{Z_{2}}(x) \ldots Q_{Z_{m}}(x) d x
$$


and

$$
\begin{aligned}
& \int_{0}^{1} Q_{Z_{1}+Z_{2}}(x) Q_{Z_{3}}(x) \ldots Q_{Z_{m}}(x) d x \leq \\
& \int_{0}^{1}\left(Q_{Z_{1}}(x)+Q_{Z_{2}}(x)\right) Q_{Z_{3}}(x) \ldots Q_{Z_{m}}(x) d x .
\end{aligned}
$$

Proof of (2.9). Let $U$ be a random variable uniformly distributed on [0,1]. Then, for any nonnegative r.v. $T, Q_{T}(U)$ has the same distribution as T. Consequently, by Lemma A1 of Berkes and Philipp (1979), one can construct random variables $T_{1}, \ldots, T_{m}$ on another probability space such that: $\left(T_{1} T_{2}, T_{3}, \ldots, T_{m}\right)$ has the same distribution as $\left(Q_{Z_{1} Z_{2}}(U), Q_{Z_{3}}(U), \ldots\right)$ and $\left(T_{1}, T_{2}\right)$ has the same law as $\left(Z_{1}, Z_{2}\right)$. So, by Lemma 1 , we have

$$
\begin{aligned}
\int_{0}^{1} Q_{Z_{1} Z_{2}}(x) Q_{Z_{3}}(x) \ldots Q_{Z_{m}}(x) d x & =\mathbb{E}\left(T_{1} T_{2} \ldots T_{m}\right) \\
& \leq \int_{0}^{1} Q_{Z_{1}}(x) Q_{Z_{2}}(x) \ldots Q_{Z_{m}}(x) d x .
\end{aligned}
$$

The proof of $(2.9 \mathrm{~b})$ is omitted.

For any $i \geq p, 2 \alpha_{i} \leq u$. So, noting that

$$
\left|f^{\prime}\left(S_{k-i}\right)-f^{\prime}\left(S_{k-i-1}\right)\right| \leq b_{2}\left|X_{k-i}\right|,
$$

we have, by Proposition 1(a),

$$
\left|\operatorname{Cov}\left(f^{\prime}\left(S_{k-i}\right)-f^{\prime}\left(S_{k-i-1}\right), X_{k}\right)\right| \leq 2 b_{2} \int_{0}^{u} \mathbb{1}_{x<2 \alpha_{i}} Q_{k-i}(x) Q_{k}(x) d x
$$

From now on, we assume that $i<p$. Let us replace $X_{k}$ by $\bar{X}_{k}$. Applying Proposition 1(a), we get that

$$
\begin{aligned}
\left|\operatorname{Cov}\left(f^{\prime}\left(S_{k-i}\right)-f^{\prime}\left(S_{k-i-1}\right), X_{k}-\bar{X}_{k}\right)\right| \leq \\
2 b_{2} \int_{0}^{u} \mathbb{1}_{x<2 \alpha_{i}} Q_{k-i}(x)\left(Q_{k}(x)-Q_{k}(u)\right) d x .
\end{aligned}
$$

Now

$$
f^{\prime}\left(S_{k-i}\right)-f^{\prime}\left(S_{k-i-1}\right)-f^{\prime \prime}\left(S_{k-i-1}\right) X_{k-i}=R_{k, i},
$$

where $R_{k, i}$ is $\mathcal{F}_{k-i}$-measurable and $\left|R_{k, i}\right| \leq b_{3} X_{k-i}^{2} / 2$. Consequently, by Proposition 1(a), we have:

$$
\left|\operatorname{Cov}\left(R_{k, i}, \bar{X}_{k}\right)\right| \leq b_{3} \int_{0}^{2 \alpha_{i}} Q_{k-i}^{2}(x) Q_{k}(x \vee u) d x .
$$

In order to estimate the term $\operatorname{Cov}\left(f^{\prime \prime}\left(S_{k-i-1}\right) X_{k-i}, \bar{X}_{k}\right)$, we introduce the decomposition below:

$$
f^{\prime \prime}\left(S_{k-i-1}\right)=\sum_{l=1}^{i-1}\left(f^{\prime \prime}\left(S_{k-i-l}\right)-f^{\prime \prime}\left(S_{k-i-l-1}\right)\right)+f^{\prime \prime}\left(S_{k-2 i}\right) .
$$


By Proposition 1(a) applied with $X=\Gamma_{k, l+i} X_{k-i}$ and $Y=X_{k}$ together with Lemma 1 and (2.9a),

$$
\left|\operatorname{Cov}\left(\Gamma_{k, l+i} X_{k-i}, \bar{X}_{k}\right)\right| \leq 2 b_{3} \int_{0}^{2 \alpha_{i}} Q_{k-i-l}(x) Q_{k-i}(x) Q_{k}(x \vee u) d x .
$$

Next, by Lemma 1 applied to independent r.v.'s,

$$
\left|\mathbb{E}\left(f^{\prime \prime}\left(S_{k-2 i}\right) X_{k-i}\right) \mathbb{E}\left(\bar{X}_{k}\right)\right| \leq b_{2} \int_{0}^{u} \mathbb{I}_{x<2 \alpha_{i}} Q_{k-i}(x)\left(Q_{k}(x)-Q_{k}(u)\right) d x
$$

(because $\mathbb{E}\left(\bar{X}_{k}\right)=\mathbb{E}\left(\bar{X}_{k}-X_{k}\right)$ and $\left.u<2 \alpha_{i}\right)$.

As a second step, we bound up $\left|\operatorname{Cov}\left(f^{\prime \prime}\left(S_{k-2 i}\right), X_{k-i} \bar{X}_{k}\right)\right|$. Clearly

$$
f^{\prime \prime}\left(S_{k-2 i}\right)=\sum_{l=i}^{p-1} \Gamma_{k, l+i}+f^{\prime \prime}\left(S_{k-i-p}\right) .
$$

Now, by Proposition 1(a) applied with $X=\Gamma_{k, l+i}, Y=X_{k-i} \bar{X}_{k}$ and (2.9a)

$$
\left|\operatorname{Cov}\left(\Gamma_{k, l+i}, X_{k-i} \bar{X}_{k}\right)\right| \leq 2 b_{3} \int_{0}^{2 \alpha_{l}} Q_{k-i-l}(x) Q_{k-i}(x) Q_{k}(x \vee u) d x .
$$

Noting that $2 \alpha_{p} \leq u<2 \alpha_{i}$, applying both (a) of Proposition 1 with $X=$ $f^{\prime \prime}\left(S_{k-i-p}\right), Y=X_{k-i} \bar{X}_{k}$ and $(2.9 \mathrm{a})$, we also get that

$$
\left|\operatorname{Cov}\left(f^{\prime \prime}\left(S_{k-i-p}\right), X_{k-i} \bar{X}_{k}\right)\right| \leq 2 b_{2} \int_{0}^{u} \mathbb{1}_{x<2 \alpha_{i}} Q_{k-i}(x) Q_{k}(u) d x .
$$

Adding the inequalities (2.10), (2.11), (2.12), (2.13), (2.14), (2.15) and (2.16) and summing on $i$ and $l$, we then get :

$$
\begin{aligned}
\mid \mathbb{E}\left(f^{\prime}\left(S_{k-1}\right) X_{k}\right) & -\sum_{i=1}^{p-1} \mathbb{E}\left(f^{\prime \prime}\left(S_{k-2 i}\right)\right) \mathbb{E}\left(X_{k-i} \bar{X}_{k}\right) \mid \leq \\
& 3 b_{2} \int_{0}^{u}\left(M_{k}(x)-Q_{k}(x)\right) Q_{k}(x) d x+ \\
& 2 b_{3} \int_{0}^{1}\left(M_{k}^{\prime}(x, u)-M_{k}(x, u) Q_{k}(x)\right) Q_{k}(x \vee u) d x .
\end{aligned}
$$

It remains to bound up

$$
D_{k}=\sum_{i=1}^{p-1} \mathbb{E}\left(f^{\prime \prime}\left(S_{k-2 i}\right)\right) \mathbb{E}\left(X_{k-i} \bar{X}_{k}\right)-\sum_{i=1}^{k-1} \mathbb{E}\left(f^{\prime \prime}\left(S_{k-1}\right)\right) \mathbb{E}\left(X_{k-i} X_{k}\right) .
$$

We first note that, by Proposition 1(a),

$$
\begin{aligned}
\sum_{i \geq p}\left|\mathbb{E}\left(f^{\prime \prime}\left(S_{k-1}\right)\right) \mathbb{E}\left(X_{k-i} X_{k}\right)\right| \leq b_{2} \sum_{i \geq p}\left|\mathbb{E}\left(X_{k-i} X_{k}\right)\right| \\
\leq b_{2} \int_{0}^{u} \sum_{i \geq p} \mathbb{I}_{x<2 \alpha_{i}} Q_{k-i}(x) Q_{k}(x) d x .
\end{aligned}
$$


Next, noting that $u<2 \alpha_{i}$ for all $i<p$ and applying Lemma 1 , we get that

$$
\begin{aligned}
& \sum_{i=1}^{p-1}\left|\mathbb{E}\left(f^{\prime \prime}\left(S_{k-1}\right)\right) \mathbb{E}\left(X_{k-i}\left(X_{k}-\bar{X}_{k}\right)\right)\right| \leq \\
& b_{2} \int_{0}^{u} \sum_{i=1}^{p-1} \mathbb{I}_{x<2 \alpha_{i}} Q_{k-i}(x)\left(Q_{k}(x)-Q_{k}(u)\right) d x .
\end{aligned}
$$

In order to bound up the last term, we still write

$$
\mathbb{E}\left(f^{\prime \prime}\left(S_{k-1}\right)-f^{\prime \prime}\left(S_{k-2 i}\right)\right) \mathbb{E}\left(X_{k-i} \bar{X}_{k}\right)=\sum_{l=1}^{2 i-1} \mathbb{E}\left(\Gamma_{k, l}\right) \mathbb{E}\left(X_{k-i} \bar{X}_{k}\right) .
$$

Both this decomposition, Lemma 1 and (2.9a) then yield :

$$
\begin{aligned}
\sum_{i=1}^{p-1} \mid \mathbb{E}\left(f^{\prime \prime}\left(S_{k-1}\right)-\right. & \left.f^{\prime \prime}\left(S_{k-2 i}\right)\right) \mathbb{E}\left(X_{k-i} \bar{X}_{k}\right) \mid \\
& \leq b_{3} \sum_{i=1}^{p-1} \sum_{l=1}^{2 i-1} \int_{0}^{2 \alpha_{i}} Q_{k-l}(x) Q_{k-i}(x) Q_{k}(x \vee u) d x \\
& \leq 2 b_{3} \int_{0}^{1} M_{k}^{\prime}(x, u) Q_{k}(x \vee u) d x
\end{aligned}
$$

Hence

$$
\left|\mathbb{E}\left(D_{k}\right)\right| \leq 2 b_{3} \int_{0}^{1} M_{k}^{\prime}(x, u) Q_{k}(x \vee u) d x+b_{2} \int_{0}^{u} M_{k}(x) Q_{k}(x \vee u) d x .
$$

(2.21) and (2.17) together with (2.7) then yield (a).

Starting from Proposition 2(a), we now prove (b). This will be done via a more tractable upper bound for $M_{k}^{\prime}(x, u)$. By the Minkowski inequality,

$$
Q_{k-i}(x) Q_{k-i-l}(x) \leq \frac{1}{2}\left(Q_{k-i}^{2}(x)+Q_{k-i-l}^{2}(x)\right) .
$$

Hence

$2 M_{k}^{\prime}(x, u) \leq \sum_{i=0}^{p-1} Q_{k-i}^{2}(x) \mathbb{1}_{x<2 \alpha_{i}} \sum_{l=0}^{p-1} \mathbb{I}_{x<2 \alpha_{l}}+\sum_{m=0}^{2 p-2} Q_{k-m}^{2}(x) \sum_{i=0}^{p-1} \mathbb{I}_{x<2\left(\alpha_{i} \wedge \alpha_{m-i}\right)}$.

Let $q(m)=m-[m / 2]$, square brackets designating the integer part, as usual. The inequality $\alpha_{i} \wedge \alpha_{m-i} \leq \alpha_{q(m)}$ lets us show that

$$
\sum_{i=0}^{p-1} \mathbb{I}_{x<2\left(\alpha_{i} \wedge \alpha_{m-i}\right)} \leq\left[\alpha^{-1}(x / 2) \wedge p\right] \alpha_{q(m)}
$$


Hence

$$
M_{k}^{\prime}(x, u) \leq \alpha^{-1}((x \vee u) / 2) \sum_{m=0}^{k-1} Q_{k-m}^{2}(x) \mathbb{I}_{x<2 \alpha_{q(m)}} .
$$

Let $\psi(x)=\inf \left(b_{2}, b_{3} x\right)$. Let the real $u$ be defined in such a way that

$$
b_{3} \alpha^{-1}\left(u^{-} / 2\right) Q_{k}\left(u^{-}\right) \geq b_{2} \geq b_{3} \alpha^{-1}(u / 2) Q_{k}(u) .
$$

With the above choice of $u$,

$$
b_{3} M_{k}^{\prime}(x, u) Q_{k}(x \vee u) \leq \sum_{m=0}^{k-1} Q_{k-m}^{2}(x) \mathbb{1}_{x<2 \alpha_{q(m)}} \psi\left(\alpha^{-1}(x / 2) Q_{k}(x)\right) .
$$

Since $\psi$ is a nondecreasing function,

$$
\begin{aligned}
& Q_{k-m}^{2}(x) \psi\left(\alpha^{-1}(x / 2) Q_{k}(x)\right) \leq \\
& Q_{k}^{2}(x) \psi\left(\alpha^{-1}(x / 2) Q_{k}(x)\right)+Q_{k-m}^{2}(x) \psi\left(\alpha^{-1}(x / 2) Q_{k-m}(x)\right) .
\end{aligned}
$$

Consequently the inequality (2.23) lets us show that

$$
\begin{array}{r}
b_{3} M_{k}^{\prime}(x, u) Q_{k}(x \vee u) \leq 2 \alpha^{-1}(x / 2) Q_{k}^{2}(x) \psi\left(\alpha^{-1}(x / 2) Q_{k}(x)\right) \\
+\sum_{m=0}^{k-1} Q_{k-m}^{2}(x) \psi\left(\alpha^{-1}(x / 2) Q_{k-m}(x)\right) \mathbb{I}_{x<2 \alpha_{q(m)}} .
\end{array}
$$

In the same way, one can prove that

$$
M_{k}(x) Q_{k}(x) \leq \alpha^{-1}(x / 2) Q_{k}^{2}(x)+\sum_{m=0}^{k-1} Q_{k-m}^{2}(x) \mathbb{I}_{x<2 \alpha_{m}} .
$$

Hence, combining Proposition 2(a) with (2.25), (2.26) and (2.22), we get Proposition 2(b).

We now finish the proof of Theorem 1 . Let $\varphi_{k}$ denote the characteristic function of $S_{k}$ and $t$ be some positive real. We now apply Proposition 2(b) to the functions $x \rightarrow \cos t x$ and $x \rightarrow \sin t x$, yielding

$$
\begin{aligned}
& \sum_{k=1}^{n}\left|\varphi_{k}(t)-\left(1-\frac{v_{k} t^{2}}{2}\right) \varphi_{k-1}(t)\right| \leq \\
& \quad 34 t^{2} \sum_{k=1}^{n} \int_{0}^{1} \alpha^{-1}(x / 2) Q_{k}^{2}(x)\left(t \alpha^{-1}(x / 2) Q_{k}(x) \wedge 1\right) d x
\end{aligned}
$$

In order to introduce the characteristic function of the normal distribution, we need to control $\left|\exp \left(-v_{k} t^{2} / 2\right)-\left(1-v_{k} t^{2} / 2\right)\right|$. By Proposition $1(\mathrm{a})$

$$
\left|v_{k}\right| \leq 4 \sum_{i=0}^{k-1} \int_{0}^{1} Q_{k}(x) Q_{k-i}(x) d x \leq 4\left(x_{k}+y_{k}\right)
$$


where

$$
x_{k}=\int_{0}^{u_{k}} Q_{k}(x) M_{k}(x) d x, \quad y_{k}=\int_{0}^{1} Q_{k}\left(x \vee u_{k}\right) \sum_{i=0}^{p_{k}-1} Q_{k-i}(x) \mathbb{I}_{x<2 \alpha_{i}} d x,
$$

$p_{k}=\alpha^{-1}\left(u_{k} / 2\right)$ and $u_{k}$ is the positive real such that

$$
t \alpha^{-1}\left(u_{k} / 2\right) Q_{k}\left(u_{k}\right) \leq 1 \leq t \alpha^{-1}\left(u_{k}^{-} / 2\right) Q_{k}\left(u_{k}^{-}\right)
$$

Let $v$ be any real and $x, y$ be positive numbers such that $|v| \leq x+y$. It is not difficult to check that

$$
\exp (v)-1-v \leq\left(x+y^{2} / 2\right) \sup (1, \exp (v)) .
$$

Applying (2.29) to $v=-v_{k}^{2} t^{2} / 2, x=2 x_{k} t^{2}$ and $y=2 y_{k} t^{2}$, we obtain :

$$
\left|\exp \left(-v_{k} t^{2} / 2\right)-\left(1-v_{k} t^{2} / 2\right)\right| \leq 2 t^{2} \sup \left(1, \exp \left(-v_{k} t^{2} / 2\right)\right)\left(x_{k}+y_{k} t^{2}\right) .
$$

Now, by (2.9a),

$$
\begin{aligned}
y_{k} t^{2} & \leq \int_{0}^{1} t^{2} Q_{k}^{2}\left(x \vee u_{k}\right) \sum_{i=0}^{p_{k}-1} \sum_{j=0}^{p_{k}-1} Q_{k-i}(x) Q_{k-j}(x) \mathbb{I}_{x<2\left(\alpha_{i} \wedge \alpha_{j}\right)} d x \\
& \leq 2 \int_{0}^{1} t^{2} Q_{k}^{2}\left(x \vee u_{k}\right) M_{k}^{\prime}\left(x, u_{k}\right) d x \\
& \leq 2 \int_{0}^{1} t Q_{k}\left(x \vee u_{k}\right) M_{k}^{\prime}\left(x, u_{k}\right) d x
\end{aligned}
$$

(because $\left.t Q_{k}\left(x \vee u_{k}\right) \leq 1 / \alpha^{-1}\left(\left(x \vee u_{k}\right) / 2\right) \leq 1\right)$. Hence, arguing as in the proof of of Proposition 2(b), we get that

$$
2 t^{2} \sum_{k=1}^{n}\left(x_{k}+y_{k} t^{2}\right) \leq 24 t^{2} \sum_{k=1}^{n} M_{3, \alpha}\left(Q_{k}, t\right) .
$$

The relations $(2.27),(2.30)$ and $(2.32)$ show that

$$
\begin{aligned}
& \sum_{k=1}^{n} \mid \exp \left(\frac{V_{k} t^{2}}{2}\right) \varphi_{k}(t)-\exp \left(\frac{V_{k-1} t^{2}}{2}\right) \varphi_{k-1}(t) \mid \\
& \leq 58 t^{2} \sup _{k \in[1, n]} \exp \left(\frac{V_{k} t^{2}}{2}\right) \sum_{k=1}^{n} M_{3, \alpha}\left(Q_{k}, t\right) \\
& \leq 58 t^{2} \exp \left(\frac{V_{n}^{*} t^{2}}{2}\right) \sum_{k=1}^{n} M_{3, \alpha}\left(Q_{k}, t\right)
\end{aligned}
$$

which concludes the proof of Theorem 1(i).

Now, under the assumption (1.3), by Proposition 2 (a),

$$
\left|\varphi_{k}(t)-\left(1-\frac{v_{k} t^{2}}{2}\right) \varphi_{k-1}(t)\right| \leq 8 \sqrt{2} t^{2} M_{3, \alpha}(Q, t) .
$$


Now, appying (2.31) and arguing as in the proof of (2.32), we get:

$$
\left|\exp \left(-v_{k} t^{2} / 2\right)-\left(1-v_{k} t^{2} / 2\right)\right| \leq 4 t^{2} M_{3, \alpha}(Q, t)\left(1+\exp \left(-v_{k} t^{2} / 2\right)\right) .
$$

Hence

$$
\begin{aligned}
\mid \exp \left(\frac{V_{k} t^{2}}{2}\right) \varphi_{k}(t)- & \exp \left(\frac{V_{k-1} t^{2}}{2}\right) \varphi_{k-1}(t) \mid \leq \\
& 4 t^{2} M_{3, \alpha}(Q, t)\left((1+2 \sqrt{2}) \exp \left(\frac{V_{k} t^{2}}{2}\right)+\exp \left(\frac{V_{k-1} t^{2}}{2}\right)\right),
\end{aligned}
$$

therefore completing the proof of (ii)

\section{Berry-Esseen type Estimates.}

Throughout, the letter $C$ is used to denote a constant (depending on the parameters) whose value may change from line to line.

Proof of Theorem 2. We start by replacing the initial random variables by three independent blocks each of length $n / 3$. In order to give an estimate of the nearness of the characteristic functions, we will prove the following lemma.

LEMma 2. Let $\left(X_{i}\right)_{i \in \mathbb{Z}}$ be a sequence of real-valued random variables satisfying the assumptions of Theorem 2 and $Q=1 \vee \sup _{i>0} Q_{i}$. Then, for any integer $l$ in $] 1, n[$ and any positive $t$,

$$
\mid \mathbb{E}\left(\exp \left(i t S_{n}\right)\right)-\mathbb{E}\left(\exp \left(i t S_{l}\right)\right) \mathbb{E}\left(\exp \left(i t\left(S_{n}-S_{l}\right)\right) \mid \leq 16 t M_{3, \alpha}(Q,|t|) .\right.
$$

Proof. We may w.l.o.g. assume $t>0$. Set

$$
\Delta=\mathbb{E}\left(\exp \left(i t S_{n}\right)\right)-\mathbb{E}\left(\exp \left(i t S_{l}\right)\right) \mathbb{E}\left(\exp \left(i t\left(S_{n}-S_{l}\right)\right)\right) .
$$

Let $u$ be defined by

$$
t \alpha^{-1}(u / 2) Q(u) \leq 1 \leq t \alpha^{-1}\left(u^{-} / 2\right) Q\left(u^{-}\right) .
$$

Let $p=\alpha^{-1}(u)$. We have :

$$
\begin{aligned}
\Delta=\sum_{k=1}^{l} & \sum_{j=1}^{p} \operatorname{Cov}\left(e^{i t S_{k}}-e^{i t S_{k-1}}, e^{i t\left(S_{n}-S_{l+j}\right)}\left(1-e^{i t X_{l+j}}\right)\right) \\
& +\sum_{k=1}^{l} \operatorname{Cov}\left(e^{i t S_{k}}-e^{i t S_{k}-1}, e^{i t\left(S_{n}-S_{l+p}\right)}\right)
\end{aligned}
$$

where $\operatorname{Cov}(X, Y)=\mathbb{E}(X Y)-\mathbb{E}(X) \mathbb{E}(Y)$. Now it follows from proposition 1 that, if $X$ and $Y$ are complex-valued random variables such that $\alpha(\sigma(X), \sigma(Y)) \leq \alpha$,

$$
|\mathbb{E}(X Y)-\mathbb{E}(X) \mathbb{E}(Y)| \leq 8 \int_{0}^{2 \alpha} Q_{X}(x) Q_{Y}(x) d x
$$


where $Q_{X}$ is defined exactly as in Proposition 1 (to prove this fact, note that the quantile functions of the imaginary part and the real part of $X$ are less than $Q_{X}$ and apply Proposition 1(a) to each of the components of the product). The above inequality and (3.3) together with the elementary inequality $\left|\exp \left(i t X_{m}\right)-1\right| \leq\left|t X_{m}\right|$ yield

$$
\begin{aligned}
\Delta & \leq 8 t^{2} \sum_{k=1}^{l} \sum_{j=1}^{p} \int_{0}^{2 \alpha_{j+k}} Q^{2}(x) d x+8 t \sum_{k=1}^{l} \int_{0}^{2 \alpha_{p+k}} Q(x) d x \\
& \leq 8 t \int_{0}^{1}\left(t \alpha^{-1}((x \vee u) / 2) Q(x \vee u)+\mathbb{1}_{x<u}\right) \alpha^{-1}(x / 2) Q^{2}(x) d x .
\end{aligned}
$$

Together with (3.1), it implies Lemma 2.

Now let us divide $n$ by $3: n=3 m+r$ for some $r$ in $\{0,1,2\}$. Set

$$
\varphi_{n, 1}=\varphi_{m}, \varphi_{n, 2}(t)=\mathbb{E}\left(e^{i t\left(S_{2 m}-S_{m}\right)}\right), \varphi_{n, 3}(t)=\mathbb{E}\left(e^{i t\left(S_{n}-S_{2 m}\right)}\right) .
$$

By Lemma 2 applied twice,

$$
\left|\varphi_{n}(t)-\prod_{i=1}^{3} \varphi_{n, i}(t)\right| \leq 32 t M_{3, \alpha}(Q,|t|) .
$$

Since $\left(X_{i}\right)_{i \in \mathbb{Z}}$ is a weakly stationary sequence verifying (1.8),

$$
\lim _{k \rightarrow \infty} v_{k}=\sigma^{2} \neq 0
$$

It follows that there exists some positive integer $n_{0}$ such that, for any $n>n_{0}$ and any $k$ in $[0, n]$,

$$
\frac{1}{2}\left(V_{n}-V_{k}\right) t^{2} \geq(n-k) \frac{\sigma^{2} t^{2}}{4}
$$

Hence, by Theorem 1(ii),

$$
\left|\varphi_{n}(t)-\exp \left(-V_{n} t^{2} / 2\right)\right| \leq 8(\sqrt{2}+1)\left(n t^{2} \wedge 4 \sigma^{-2}\right) M_{3, \alpha}(Q,|t|) .
$$

Let $\kappa_{n, i}=-\varphi_{n, i}^{(2)}(0)$. It follows from (3.8) and (3.9) that

$$
\left|\varphi_{n, i}(t)-\exp \left(-\kappa_{n, i} t^{2} / 2\right)\right| \leq C\left(n t^{2} \wedge \sigma^{-2}\right) M_{3, \alpha}(Q,|t|)
$$

for any $n \geq 3 n_{0}+3$. For sake of brevity, we set $M_{3, \alpha}(Q,|t|)=M_{3, \alpha}(t)$. Both (3.10) and (3.8) applied with $k=0$ imply that

$$
\begin{aligned}
& \quad\left|\prod_{i=1}^{3} \varphi_{n, i}(t)-\prod_{i=1}^{3} \exp \left(-\kappa_{n, i} t^{2} / 2\right)\right| \leq \\
& \quad C\left(n t^{2} \wedge 1\right)\left(M_{3, \alpha}^{3}(t)+M_{3, \alpha}(t) \exp \left(-m \sigma^{2} t^{2} / 4\right)\right),
\end{aligned}
$$


where $C$ is some positive constant depending only on $\sigma$. Let $V=\sum_{i=1}^{3} \kappa_{n, i}$ (note that $V \geq n \sigma^{2} / 2$ for $n$ large enough). (3.6) and (3.11) let us show that

$$
\begin{aligned}
\mid \varphi_{n}(t)- & \exp \left(-V t^{2} / 2\right) \mid \leq \\
& C M_{3, \alpha}(t)\left(\inf \left(1, n t^{2}\right)\left(\exp \left(-m \sigma^{2} t^{2} / 4\right)+M_{3, \alpha}^{2}(t)\right)+t\right) .
\end{aligned}
$$

Let $F_{n}$ denote the distribution function of $S_{n}$. By (3.12) and Theorem 2, p. 109 in Petrov (1975),

$$
\begin{aligned}
& \sup _{x \in \mathbb{R}}\left|F_{n}(x)-\Psi(x / \sqrt{V})\right| \leq C\left(\frac{1}{T \sqrt{V}}+T M_{3, \alpha}(T)+\right. \\
& \left.\int_{0}^{T}\left(n t^{2} \wedge 1\right)\left(M_{3, \alpha}(t) \exp \left(-m \sigma^{2} t^{2} / 4\right)+M_{3, \alpha}^{3}(t)\right) \frac{d t}{t}\right) .
\end{aligned}
$$

Now, if $M_{3, \alpha}(Q)<\infty, M_{3, \alpha}(t) \leq t M_{3, \alpha}(Q)$. Hence, by (3.13),

$$
\sup _{x \in \mathbb{R}}\left|F_{n}(x)-\Psi(x / \sqrt{V})\right| \leq C\left(\frac{1}{T \sqrt{V}}+T^{2}+T^{3}+\frac{1}{\sigma \sqrt{n}}\right) .
$$

(3.14) applied with $T=n^{-1 / 6}$ then yields :

$$
\sup _{x \in \mathbb{R}}\left|F_{n}(x)-\Psi(x / \sqrt{V})\right|=O\left(n^{-1 / 3}\right) .
$$

In order to prove (i), we note that $M_{3, \alpha}(t)=O\left(t^{\delta}\right)$ under assumption (1.9) [see Appendix]. Hence, by (3.13),

$$
\sup _{x \in \mathbb{R}}\left|F_{n}(x)-\Psi(x / \sqrt{V})\right| \leq C\left(\frac{1}{T \sqrt{V}}+T^{3 \delta \wedge(1+\delta)}+\left(n \sigma^{2}\right)^{-\delta / 2}\right) .
$$

First, if $\delta \leq 1 / 2,3 \delta \leq 1+\delta$, and applying (3.16) with $T M_{2, \alpha}=n^{-1 /(6 \delta+2)}$, we get :

$$
\sup _{x \in \mathbb{R}}\left|F_{n}(x)-\Psi(x / \sqrt{V})\right|=O\left(n^{-\delta / 2} \vee n^{-3 \delta /(6 \delta+2)}\right)=O\left(n^{-\delta / 2}\right) .
$$

Secondly, if $\delta>1 / 2,3 \delta>1+\delta$, and applying (3.16) with $T M_{2, \alpha}=$ $n^{-1 /(4+2 \delta)}$, we get :

$$
\sup _{x \in \mathbb{R}}\left|F_{n}(x)-\Psi(x / \sqrt{V})\right|=O\left(n^{-\delta / 2} \vee n^{-(1+\delta) /(4+2 \delta)}\right) .
$$

To prove (iii), we note that, since $M_{3, \alpha}(t)$ is a nondecreasing function of $t,(3.13)$ yields

$$
\begin{gathered}
\sup _{x \in \mathbb{R}}\left|F_{n}(x)-\Psi(x / \sqrt{V})\right| \leq C\left(\frac{1}{T \sqrt{V}}+T M_{3, \alpha}(T)+M_{3, \alpha}\left(n^{-1 / 2}\right)\right. \\
\left.+\int_{1 / \sqrt{n}}^{T}\left(M_{3, \alpha}(t) \exp \left(-m \sigma^{2} \frac{t^{2}}{4}\right)+M_{3, \alpha}^{3}(t)\right) \frac{d t}{t}\right)
\end{gathered}
$$


for any $T \geq n^{-1 / 2}$. Under the assumptions of (iii), some elementary calculations let us show that $M_{3, \alpha}(t)=O\left(|\log t|^{-p}\right)$ [see Appendix], which together with (3.19) and the elementary inequality

$$
\int_{n^{-1 / 2}}^{T} M_{3, \alpha}(t) \exp \left(-m \sigma^{2} \frac{t^{2}}{4}\right) \frac{d t}{t} \leq C(\log n)^{-p}
$$

ensures that

$$
\begin{aligned}
& \sup _{x \in \mathbb{R}}\left|F_{n}(x)-\Psi(x / \sqrt{V})\right| \leq \\
& \quad C\left(\frac{1}{T \sqrt{V}}+T M_{3, \alpha}(T)+|\log T|^{-3 p} \log \left(n T^{2}\right)+|\log n|^{-p}\right) .
\end{aligned}
$$

Setting $T=n^{-1 / 2}(\log n)^{p}$ in $(3.21)$, we then get :

$$
\sup _{x \in \mathbb{R}}\left|F_{n}(x)-\Psi(x / \sqrt{V})\right|=O\left((\log n)^{-p}\right) .
$$

Taking into accounts $(3.15),(3.18)$ and $(3.22)$, it only remains to prove that

$$
\sup _{x \in \mathbb{R}}|\Psi(x \sigma \sqrt{n})-\Psi(x \sqrt{V})|=O\left(M_{3, \alpha}\left(n^{-1 / 2}\right)\right) .
$$

To prove (3.23), it is sufficient to use the estimate

$$
\left|\sqrt{\frac{V}{n \sigma^{2}}}-1\right|=O\left(M_{3, \alpha}\left(n^{-1 / 2}\right)\right)
$$

and standard calculations on the Gaussian distribution function. Now

$$
\left|\sqrt{\frac{V}{n \sigma^{2}}}-1\right| \leq \frac{1}{n \sigma^{2}}\left|V-n \sigma^{2}\right| \leq \frac{3}{n \sigma^{2}} \sum_{i \in \mathbb{Z}}(|i| \wedge n)\left|\operatorname{Cov}\left(X_{0}, X_{i}\right)\right| .
$$

Since $Q \geq 1$, we have, by Proposition 1(a),

$$
\begin{aligned}
\frac{1}{n} \sum_{i \in \mathbb{Z}}(|i| \wedge n)\left|\operatorname{Cov}\left(X_{0}, X_{i}\right)\right| & \leq \frac{4}{n} \int_{0}^{1} \alpha^{-1}(x / 2) Q^{2}(x)\left[\alpha^{-1}(x / 2) Q(x) \wedge n\right] d x \\
& \leq 4 M_{3, \alpha}(1 / n)
\end{aligned}
$$

which, together with (3.25), implies (3.24).

\section{Moment inequalities For STRONGLY MiXing SEQUENCES.}

In this section, we derive Rosenthal type inequalities for moments of partial sums from Proposition 2. These inequalities generalize the moment inequalities of Rio (1994) to nonstationary sequences or stationary sequences in the degenerate case. The main interest of these inequalities 
is that they give the right bound for integrated moments of nonparametric estimations (see for example Doukhan and Portal (1983b) and Doukhan (1991)). These inequalities improve the previous inequalities of Doukhan and Portal (1983b), Utev (1985) and Yokoyama (1980). We refer the reader to Doukhan (1994) for a detailed survey of the previous moment inequalities. In order to state these moment inequalities, we need some notations.

Definition 4. For any nondecreasing function $\phi: \mathbb{R}^{+} \rightarrow \mathbb{R}^{+}$, we set

$$
M_{\phi, \alpha, n}(Q)=\int_{0}^{1 / 2} \frac{\phi\left(\left[\alpha^{-1}(x / 2) \wedge n\right] Q(x)\right)}{\left[\alpha^{-1}(x / 2) \wedge n\right]} d x
$$

If $\phi(x)=x^{r}$, we set $M_{\phi, \alpha, n}(Q)=M_{r, \alpha, n}(Q)$.

Theorem 3. Let $\left(X_{i}\right)_{i \in \mathbb{Z}}$ be a strongly mixing sequence of real-valued random variables with finite variance and mean zero. Let $s_{n}$ be the nonnegative real defined by

$$
s_{n}^{2}=\sum_{i=1}^{n} \sum_{j=1}^{n}\left|\operatorname{Cov}\left(X_{i}, X_{j}\right)\right|
$$

Then, for any $\phi$ in $\Phi$

$$
\begin{aligned}
\mathbb{E}\left(\phi\left(\left|S_{n}\right|\right)\right) & \leq \frac{1}{2} s_{n}^{2} \phi^{\prime \prime}\left(s_{n}\right)+24 \sum_{k=1}^{n} M_{x^{2} \phi^{\prime \prime}, \alpha}\left(Q_{k}\right) \\
& \leq 3 \phi\left(s_{n}\right)+144 \sum_{k=1}^{n} M_{\phi, \alpha, n}\left(Q_{k}\right) .
\end{aligned}
$$

Suppose that $\left(X_{i}\right)_{i \in \mathbb{Z}}$ is a stationary sequence. Let $\sigma_{n}$ be the nonnegative real defined by $\sigma_{n}^{2}=\operatorname{Var} S_{n}$. Then

$$
\mathbb{E}\left(\phi\left(\left|S_{n}\right|\right)\right) \leq 3 \sqrt{2} \phi\left(\sigma_{n}\right)+180 n M_{\phi, \alpha, n}\left(Q_{0}\right)
$$

Suppose furthermore that $\left(X_{i}\right)_{i \in \mathbb{Z}}$ satisfies (1.3). Let $\sigma^{2}=\sum_{t \in \mathbb{Z}} \operatorname{Cov}\left(X_{0}, X_{t}\right)$. Then

$$
\mathbb{E}\left(\phi\left(\left|S_{n}\right|\right)\right) \leq 3 \sqrt{2} \phi(\sigma \sqrt{n})+180 n M_{\phi, \alpha}\left(Q_{0}\right)
$$

Application 3. Let us compare Theorem 3(a) with the inequalities of Rio (1994) and Yokoyama (1980). Clearly we may assume that $X_{i}=0$ for any $i \notin[1, n]$. Consequently we can take $\alpha_{k}=0$ for any $k \geq n$ in Theorem 3(a). When $\phi(x)=x^{r}$ for some $\left.\left.r \in\right] 2,3\right]$ (note that Theorem 3 does not cover the case $r>3$ ), Theorem $3($ a) yields:

$$
\mathbb{E}\left(\left|S_{n}\right|^{r}\right) \leq 3 s_{n}^{r / 2}+144 \sum_{k=1}^{n} \int_{0}^{1}\left[\alpha^{-1}(x / 2) \wedge n\right]^{r-1} Q_{k}^{r}(x) d x .
$$

By contrast Theorem 1 in Rio (1994) holds for any $r>2$. However, this theorem needs the stationarity and the more restrictive definition (1.2) of the strong mixing coefficients. 
Let us compare Theorem 3 and Theorem 1 in Rio (1994) with the Marcinkiewicz-Zygmund type inequalities of Yokohama (1980). Some elementary calculations show that

$$
\int_{0}^{1}\left[\alpha^{-1}(x / 2) \wedge n\right]^{r-1} Q_{k}^{r}(x) d x \leq \sum_{l=0}^{n-1}(l+1)^{r-2} \int_{0}^{2 \alpha_{l}} Q_{k}^{r}(x) d x .
$$

Hence, by Proposition 1(b) and Theorem 3(a), the Marcinkiewicz-Zygmund inequality $\mathbb{E}\left(\left|S_{n}\right|^{r}\right)=O\left(n^{r / 2}\right)$ holds if there exists some positive constant $C$ such that

$$
\frac{1}{n} \sum_{k=1}^{n} \int_{0}^{2 \alpha_{l}} Q_{k}^{r}(x) d x \leq C(l+1)^{-r / 2} \text { for any } l \in[1, n] .
$$

Consequently, in the stationary case (b) of Theorem $3(2<r \leq 3)$ or Theorem 1 in Rio (1994) (with the definition (1.2) of the mixing coefficients) yield $\mathbb{E}\left(\left|S_{n}\right|^{r}\right)=O\left(n^{r / 2}\right)$ under the mixing condition

$$
\int_{0}^{2 \alpha_{l}} Q_{0}^{r}(x) d x=O\left(l^{-r / 2}\right) \text { as } l \rightarrow \infty
$$

Hence $\mathbb{E}\left(\left|S_{n}\right|^{r}\right)=O\left(n^{r / 2}\right)$ in the bounded case if $\alpha_{l}=O\left(l^{-r / 2}\right)$, while Theorem 2 of Yokoyama (1980) needs the stronger summability condition $\sum_{l>0} l^{r / 2-1} \alpha_{l}<\infty$.

In the unbounded case, the Marcinkiewicz-Zygmund inequality holds under the tail condition

$$
\mathbb{P}\left(\left|X_{0}\right|>u\right)=O\left(u^{-r-\delta}\right) \text { for some } \delta>0
$$

(this condition is weaker than the moment condition of Yokoyama) as soon as $\alpha_{l}=O\left(l^{-r(\delta+r) /(2 \delta)}\right)$, which is a weaker condition than Yokohama's mixing condition $\sum_{l>0} l^{r / 2-1} \alpha_{l}^{\delta /(r+\delta)}<\infty$.

Proof. We start by proving Theorem 3 in the case $\phi^{\prime \prime}(0)=0$. Suppose furthermore that $\phi$ satisfies the additional condition

$$
\lim _{x \rightarrow+\infty} \phi^{(3)}(x)=0
$$

Let $\nu$ be the Stieltjes measure of $-\phi^{(3)}$, i.e. $\nu=-d \phi^{(3)}$. Let the function $g: \mathbb{R}^{+} \times \mathbb{R} \rightarrow \mathbb{R}^{+}$be defined by:

$$
6 g(t, x)=x^{3} \mathbb{1}_{x \leq t}+\left(3 t(x-t)^{2}+3 t^{2}(x-t)+t^{3}\right) \mathbb{1}_{x>t}
$$

for any $x \geq 0$ and $g(t,-x)=g(t, x)$. Clearly the following equality holds:

$$
\phi(x)=\int_{0}^{\infty} g(t, x) \nu(d t) .
$$


Hence, by the Fubini-Tonelli Theorem

$$
\mathbb{E}\left(\phi\left(\left|S_{n}\right|\right)=\int_{0}^{\infty} \mathbb{E}\left(g\left(t, S_{n}\right)\right) \nu(d t)\right.
$$

Now $x \rightarrow g(t, x)$ is a two times continuously differentiable even function and $g_{x x}^{\prime \prime}(t, x)=|x| \wedge t$. Hence we may apply Proposition $2(\mathrm{~b})$ with $b_{2}=t$ and $b_{3}=1$, yielding :

$$
\begin{aligned}
\mathbb{E}\left(g\left(t, S_{n}\right)\right) \leq & \sum_{k=1}^{n}\left(\frac{v_{k}}{2} \mathbb{E}\left(t \wedge\left|S_{k-1}\right|\right)+\right. \\
& \left.24 \int_{0}^{1} \alpha_{n}^{-1}(x / 2) Q_{k}^{2}(x)\left(\alpha_{n}^{-1}(x / 2) Q_{k}(x) \wedge t\right) d x\right),
\end{aligned}
$$

where $\alpha_{n}^{-1}(y)=\alpha^{-1}(y) \wedge n$. By the Lebesgue derivation theorem,

$$
\phi^{\prime \prime}(x)=\int_{0}^{\infty}(t \wedge|x|) \nu(d t) .
$$

Hence, integrating (4.5) with respect to the measure $\nu$, we get that

$$
\mathbb{E}\left(\phi\left(\left|S_{n}\right|\right)\right) \leq \sum_{k=1}^{n} \frac{v_{k}}{2} \mathbb{E}\left(\phi^{\prime \prime}\left(\left|S_{k-1}\right|\right)\right)+24 \sum_{k=1}^{n} M_{x^{2} \phi^{\prime \prime}, \alpha, n}\left(Q_{k}\right) .
$$

Noting then that $x \rightarrow \phi^{\prime \prime}(\sqrt{x})$ is a nondecreasing concave function, we obtain:

$$
\sum_{k=1}^{n} v_{k} \mathbb{E}\left(\phi^{\prime \prime}\left(\left|S_{k-1}\right|\right)\right) \leq \sum_{k=1}^{n}\left|v_{k}\right| \phi^{\prime \prime}\left(\sqrt{V_{k-1}}\right) \leq \sum_{k=1}^{n}\left|v_{k}\right| \phi^{\prime \prime}\left(\max _{k \in[1, n]} \sqrt{V_{k}}\right) .
$$

To prove (a), we note that $V_{k} \leq s_{n}^{2}$ and $\left|v_{1}\right|+\cdots+\left|v_{n}\right| \leq s_{n}^{2}$. It follows that

$$
\sum_{k=1}^{n} v_{k} \mathbb{E}\left(\phi^{\prime \prime}\left(\left|S_{k-1}\right|\right)\right) \leq s_{n}^{2} \phi^{\prime \prime}\left(s_{n}\right)
$$

Now, by the Taylor integral formula,

$$
\phi(x)=x^{2} \int_{0}^{1}(1-t) \phi^{\prime \prime}(t x) d t \geq x^{2} \phi^{\prime \prime}(x) \int_{0}^{1} t(1-t) d t=\frac{1}{6} x^{2} \phi^{\prime \prime}(x) .
$$

Together with (4.6) and (4.8), it implies Theorem 3(a).

Let us prove (b). In the stationary case, some elementary calculations show that

$$
v_{k}=n^{-1} \sigma_{n}^{2}+2 \sum_{t=1}^{n-1} \operatorname{Cov}\left(X_{0}, X_{t}\right)+2 \sum_{t=k}^{n-1} \operatorname{Cov}\left(X_{0}, X_{t}\right) .
$$


It follows that

$$
\left|v_{k}\right| \leq n^{-1} \sigma_{n}^{2}+2 n^{-1} \sum_{t=1}^{n-1} t\left|\operatorname{Cov}\left(X_{0}, X_{t}\right)\right|+2 \sum_{t=k}^{n-1}\left|\operatorname{Cov}\left(X_{0}, X_{t}\right)\right| .
$$

Hence, summing on $k$ and applying Proposition 1(a), we get that

$$
\begin{aligned}
\max _{k \in[1, n]}\left|V_{k}\right| & \leq \sum_{k=1}^{n}\left|v_{k}\right| \leq \sigma_{n}^{2}+4 \sum_{t=1}^{n-1} t\left|\operatorname{Cov}\left(X_{0}, X_{t}\right)\right| \\
& \leq \sigma_{n}^{2}+4 \int_{0}^{1}\left[\alpha^{-1}(x / 2) \wedge n\right]^{2} Q_{0}^{2}(x) d x
\end{aligned}
$$

Let

$$
R_{n}(x)=2\left[\alpha^{-1}(x / 2) \wedge n\right] Q_{0}(x) \text { and } I_{n}=\int_{0}^{1} R_{n}^{2}(x) d x .
$$

Using the concavity of $\phi^{\prime \prime}$, one can prove that

$$
y \phi^{\prime \prime}(y) \leq 2 \phi^{\prime}(y) \text {. }
$$

Hence, by (4.7) and (4.11)

$\sum_{k=1}^{n} v_{k} \mathbb{E}\left(\phi^{\prime \prime}\left(\left|S_{k-1}\right|\right)\right) \leq\left(I_{n}+\sigma_{n}^{2}\right) \phi^{\prime \prime}\left(\sqrt{I_{n}+\sigma_{n}^{2}}\right) \leq 2 \sqrt{I_{n}+\sigma_{n}^{2}} \phi^{\prime}\left(\sqrt{I_{n}+\sigma_{n}^{2}}\right)$.

Since $\phi^{\prime \prime}$ is nondecreasing, the function $x \rightarrow \sqrt{x} \phi(\sqrt{x})$ is convex. It follows that

$$
2 \sqrt{I_{n}+\sigma_{n}^{2}} \phi^{\prime}\left(\sqrt{I_{n}+\sigma_{n}^{2}}\right) \leq \sqrt{2 I_{n}} \phi^{\prime}\left(\sqrt{2 I_{n}}\right)+\sigma_{n} \sqrt{2} \phi^{\prime}\left(\sigma_{n} \sqrt{2}\right)
$$

Now, by Jensen's inequality,

$$
\sqrt{2 I_{n}} \phi^{\prime}\left(\sqrt{2 I_{n}}\right) \leq \sqrt{2} \int_{0}^{1} R_{n}(x) \phi^{\prime}\left(R_{n}(x) \sqrt{2}\right) d x .
$$

Now, from (4.12) the function $x \rightarrow x^{-2} \phi^{\prime}(x)$ is nonincreasing. Furthermore, integrating (4.12), we get that $x \phi^{\prime}(x) \leq 3 \phi(x)$. Hence

$$
\sqrt{2 I_{n}} \phi^{\prime}\left(\sqrt{2 I_{n}}\right) \leq 48 \sqrt{2} \int_{0}^{1} \phi\left(\left[\alpha^{-1}(x / 2) \wedge n\right] Q_{0}(x)\right) d x
$$

and

$$
\sigma_{n} \sqrt{2} \phi^{\prime}\left(\sigma_{n} \sqrt{2}\right) \leq 6 \sqrt{2} \phi\left(\sigma_{n}\right)
$$

Together with (4.13), it implies that

$$
\sum_{k=1}^{n} v_{k} \mathbb{E}\left(\phi^{\prime \prime}\left(\left|S_{k-1}\right|\right)\right) \leq 6 \sqrt{2} \phi\left(\sigma_{n}\right)+48 n \sqrt{2} M_{\phi, \alpha, n}\left(Q_{0}\right)
$$


The relations (4.6), (4.9) and (4.18) imply then Theorem 3(b).

To prove (c), we note that

$$
\left|v_{k}\right| \leq \sigma^{2}+2 \sum_{t \geq k}\left|\operatorname{Cov}\left(X_{0}, X_{t}\right)\right|
$$

which implies that

$$
\begin{aligned}
\max _{k \in[1, n]}\left|V_{k}\right| & \leq \sum_{k=1}^{n}\left|v_{k}\right| \leq n \sigma^{2}+2 \sum_{t>0}(t \wedge n)\left|\operatorname{Cov}\left(X_{0}, X_{t}\right)\right| \\
& \leq n \sigma^{2}+2 \int_{0}^{1}\left[\alpha^{-1}(x / 2) \wedge n\right] \alpha^{-1}(x / 2) Q_{0}^{2}(x) d x .
\end{aligned}
$$

Now, arguing as in the proof of (b),

$$
\begin{aligned}
& \sum_{k=1}^{n} v_{k} \mathbb{E}\left(\phi^{\prime \prime}\left(\left|S_{k-1}\right|\right)\right) \leq 6 \sqrt{2} \phi(\sigma \sqrt{n})+ \\
& 24 \int_{0}^{1} \phi\left(\sqrt{\left[\alpha^{-1}(x / 2) \wedge n\right] \alpha^{-1}(x / 2)} Q_{0}(x)\right) d x .
\end{aligned}
$$

Noting that the function $x \rightarrow x^{-2} \phi(x)$ is nondecreasing, we obtain:

$$
\phi\left(\sqrt{\left[\alpha^{-1}(x / 2) \wedge n\right] \alpha^{-1}(x / 2)} Q_{0}(x)\right) \leq \frac{\left[\alpha^{-1}(x / 2) \wedge n\right]}{\alpha^{-1}(x / 2)} \phi\left(\alpha^{-1}(x / 2) Q_{0}(x)\right) .
$$

Hence

$$
\sum_{k=1}^{n} v_{k} \mathbb{E}\left(\phi^{\prime \prime}\left(\left|S_{k-1}\right|\right)\right) \leq 6 \sqrt{2} \phi(\sigma \sqrt{n})+24 n M_{\phi, \alpha}\left(Q_{0}\right)
$$

and from now on, the end of the proof uses the same arguments as in the proof of (b).

Next we can get rid of the additional condition (4.1) by noting that, for any function $\phi$ satisfying $\phi^{\prime \prime}(0)=0$ and the assumptions of Theorem 3 , there exists a nondecreasing sequence of functions $\left(\phi_{n}\right)_{n>0}$ satisfying (4.1) and the same conditions, such that $\phi=\lim _{n} \uparrow \phi_{n}$. Hence the Beppo-Levi lemma yields the result. Finally, if $\phi^{\prime \prime}(0) \neq 0$, one can write

$$
\phi(x)=\left(\phi(x)-x^{2} \phi^{\prime \prime}(0) / 2\right)+x^{2} \phi^{\prime \prime}(0) / 2 \text {. }
$$

It is then sufficient to apply the above result to $\phi_{0}(x)=\phi(x)-x^{2} \phi^{\prime \prime}(0) / 2$. 
APPENDIX : UPPER BOUNDS ON THE TRUNCATED MOMENTS.

In this appendix, we use the notations of section 1.

Lemma A.1. Let $\phi$ be some element of $\Phi$ such that $M_{\phi, \alpha}(Q)<\infty$. Then

$$
M_{3, \alpha}(Q,|t|)=M_{3, \alpha}(t) \leq \frac{M_{\phi, \alpha}(Q)}{t^{2} \phi(1 / t)} .
$$

Assume now that $\Lambda_{2+\delta, \alpha}(Q)<+\infty$ for some $\delta$ in $] 0,1[$. Then

$$
M_{3, \alpha}(t)=O\left(t^{\delta}\right) \text { as } t \rightarrow 0 .
$$

Proof. For any $\phi$ in $\Phi$ and any $x \leq 1 / t$,

$$
t x \leq \frac{\phi(x)}{t^{2} x^{2} \phi(1 / t)}
$$

Applying this inequality to $x=\alpha^{-1}(y / 2) Q(y) \wedge(1 / t)$ and noting that $x \rightarrow$ $x^{-2} \phi(x)$ is nondecreasing, we get that

$$
\left(\alpha^{-1}(y / 2) Q(y)\right)^{2}\left(t \alpha^{-1}(y / 2) Q(y) \wedge 1\right) \leq \frac{\phi\left(\alpha^{-1}(y / 2) Q(y)\right)}{t^{2} \phi(1 / t)},
$$

which implies (A.1).

Now, let $\mu$ be the positive measure on $[0,1 / 2]$ defined by

$$
\mu=\mathbb{I}_{[0,1 / 2]}(x) \frac{d x}{\alpha^{-1}(x / 2)} .
$$

With the above notations,

$$
M_{3, \alpha}(t)=\int_{0}^{1} M^{2}(x)(t M(x) \wedge 1) \mu(d x) .
$$

Consequently (A.2) holds if

$$
\limsup _{t \rightarrow+\infty} t^{2+\delta} \mu\{x>0: M(x)>t\}<\infty .
$$

Setting $t=\alpha^{-1}(u / 2) Q(u)$, we get that this inequality holds if

$$
\limsup _{u \rightarrow 0^{+}}\left(\alpha^{-1}(u) Q(u)\right)^{2+\delta} \int_{0}^{u} \frac{d x}{\alpha^{-1}(x)}<\infty,
$$

which is a weaker condition than $\Lambda_{2+\delta, \alpha}(Q)<+\infty$. 


\section{REFERENCES}

BAss, J., (1955), Sur la compatibilité des fonctions de répartition. C.R. Acad. Sci. Paris. 240 839-841.

Bergström, H., (1972), On the convergence of sums of random variables in distribution under mixing condition. Periodica math. Hungarica. 2 173-190

Berkes, I. and PhilipP, W., (1979), Approximation theorems for independent and weakly dependent random vectors. Ann. Probab. 7 29-54.

Bolthausen, E., (1980), The Berry-Esseen theorem for functionals of discrete Markov chains. Z. Wahrsch. verw. Gebiete. 54 59-73.

Bolthausen, E., (1982), The Berry-Esseen theorem for strongly mixing Harris recurrent Markov chains. Z. Wahrsch. verw. Gebiete 60 283-289.

Bulinskit, A. V. and Doukhan, P., (1990), Vitesse de convergence dans le théorème de limite centrale pour des champs mélangeants satisfaisant des hypothèses de moment faibles. C. R. Acad. Sci. Paris, Série I, 311 801-805.

Davydov, Yu. A., (1968), Convergence of distributions generated by stationary stochastic processes. Theory Probab. Appl. 13 691-696.

Doukhan, P., (1991), Consistency of $\delta$-estimates for a regression or a density in a dependent framework. Séminaire d'Orsay 1989-1990: Estimation fonctionnelle. Prépublication mathématique de l'université de Paris-Sud.

Doukhan, P., (1994), Mixing. Properties and Examples. Lecture Notes in Statistics 85. Springer, New York.

Doukhan, P., León, J. and Portal, F., (1984), Vitesse de convergence dans le théorème central limite pour des variables aléatoires mélangeantes à valeurs dans un espace de Hilbert. C. R. Acad. Sci. Paris Série I, 298 305-308.

Doukhan, P., León, J. and Portal, F., (1985), Calcul de la vitesse de convergence dans le théorème central limite vis à vis des distances de Prohorov, Dudley et Lévy dans le cas de variables aléatoires dépendantes. Probab. Math. Stat. 6 $19-27$.

Doukhan, P., Massart, P. and Rio, E., (1994), The functional central limit Theorem for strongly mixing processes. Annales inst. H. Poincaré Probab. Statist. 30 $63-82$.

Doukhan, P. and Portal, F., (1983a), Principe d'invariance faible avec vitesse pour un processus empirique dans un cadre multidimensionnel et fortement mélangeant. C. R. Acad. Sci. Paris, Série I, 297 505-508.

Doukhan, P. and Portal, F., (1983b), Moments de variables aléatoires mélangeantes. C. R. Acad. Sci. Paris, Série I, 297 129-132.

Doukhan, P. and Portal, F., (1987), Principe d'invariance faible pour la fonction de répartition empirique dans un cadre multidimensionnel et mélangeant. Probab. Math. Stat. 8 117-132.

Esseen, C., (1945), Fourier analysis of distribution functions. A mathematical study of the Laplace-Gaussian law. Acta Math. 77 1-125.

FrÉchet, M., (1951), Sur les tableaux de corrélation dont les marges sont données. Annales de l'université de Lyon, Sciences, section A. 14 53-77.

Fréchet, M., (1957), Sur la distance de deux lois de probabilité. C. R. Acad. Sci. Paris $244689-692$.

Gordin, M. I., (1969), The central limit theorem for stationary processes. Soviet Math. Dokl. $101174-1176$.

Götze, F. and Hipp, C., (1983), Asymptotic expansions for sums of weakly dependent random vectors. $Z$. Wahrsch. verw. Gebiete. 64 211-239.

Hall, P. and Heyde, C. C., (1980), Martingale limit theory and its applications, Academic Press.

IBRAgimov, I. A., (1962), Some limit theorems for stationary processes. 7 349-382.

Ibragimov, I. A. and Linnik, Y. V., (1971), Independent and stationary sequences of random variables, Wolters-Noordhoff, Amsterdam.

KRIEGER, H. A., (1984), A new look at Bergström's theorem on convergence in distribution for sums of dependent random variables. Israel J. Math. 47 32-64. 
LindeBerg, J. W., (1922), Eine neue Herleitung des Exponentialgezetzes in der Wahrscheinlichkeitsrechnung. Mathematische Zeitschrift. 15 211-225.

Peligrad, M., (1995), On the asymptotic normality of sequences of weak dependent random variables. To appear in J. of Theoret. Probab.

Peligrad, M. and Utev, S., (1994), Central limit theorem for stationary linear processes. Preprint.

Petrov, V. V., (1975), Sums of independent random variables. Springer, Berlin.

Rio, E., (1993), Covariance inequalities for strongly mixing processes. Annales inst. H. Poincaré Probab. Statist. 29 587-597.

Rio, E., (1994), Inégalités de moments pour les suites stationnaires et fortement mélangeantes. C. R. Acad. Sci. Paris, Série I. 318 355-360.

Rosenblatt, M., (1956), A central limit theorem and a strong mixing condition. Proc. Natl. Acad. Sci. U.S.A. 42 43-47.

Rosenthal, H. P., (1970), On the subspaces of $L^{p},(p>2)$ spanned by sequences of independent random variables. Israel J. Math. 8 273-303.

SAMUR, J. D., (1984), Convergence of sums of mixing triangular arrays of random vectors with stationary rows. Ann. Probab. 12 390-426.

STEIN, C., (1972), A bound on the error in the normal approximation to the distribution of a sum of dependent random variables. Proc. Sixth Berkeley Symp. Math. Statist, and Prob. II 583-602.

TikhomiRov, A. N., (1980), On the convergence rate in the central limit theorem for weakly dependent random variables. Theor. Probab. Appl. 25 790-809.

UTEv, S., (1985), Inequalities and estimates of the convergence rate for the weakly dependent case. Proceedings of the institute of mathematics Novosibirsk. Limit theorems for sums of random variables. Adv. in Probab. Theory. 73-114. Editor A. A. Borovkov. Optimization Software, Inc. New York.

Yokoyama, R., (1980), Moment bounds for stationary mixing sequences. Z. Wahrsch. verw. Gebiete. 52 45-57.

YuRINSkiI, V. V., (1977), On the error of the Gaussian approximation for convolutions. Theory Probab. Appl. 22 236-247.

Emmanuel Rio, URA 0743 CNRS, Université Paris Sud, BÂt. 425, MathémaTIQUE, F-91405 ORSAY CEDEX 